\title{
Spectral and Scattering Theory for a Class of Strongly Oscillating Potentials
}

\author{
Monique Combescure \\ Laboratoire de Physique Théorique et Hautes Energies *, \\ Université de Paris-Sud, F-91405 Orsay, France
}

\begin{abstract}
Following a recent investigation by Pearson [23] on scattering theory for some class of oscillating potentials, we consider the Schrödinger operator in $L^{2}\left(\mathbb{R}^{n}\right)$ given by: $H=-e^{-U} \nabla \cdot e^{2 U} \nabla e^{-U}+e^{-2 U}(F+(\nabla \cdot Q))$. Here $U$ and $F$ are real functions of $x$, and $Q$ is a $\mathbb{R}^{n}$-valued function of $x$, such that:

(1) $U$ is bounded, and the local singularities of $F$ and $Q^{2}$ are controlled in a suitable sense by the kinetic energy,

(2) $U, Q$, and $F$ tend to zero at infinity faster than $|x|^{-1}$. We define $H$ by a method of quadratic forms and derive the usual results of scattering theory, namely: the absolutely continuous spectrum is $[0, \infty)$ and the singular continuous spectrum is empty, the wave operators exist and are asymptotically complete. This enlarges the class of already studied strongly oscillating potentials that give rise to the scattering and spectral properties mentioned above.
\end{abstract}

\section{Introduction}

The spectral and scattering theory of the Schrödinger operator $H=H_{0}+V$ where $H_{0}=-\Delta$, and $V$ is a real-valued function of $x\left(x \in \mathbb{R}^{n}\right)$, has been shown to extend to potentials of the form $V=(\nabla \cdot W)$ where $W$ is a $\mathbb{R}^{n}$-valued function of $x$, and where all assumptions on local behaviour and decrease at infinity are made on $W^{2}$ instead of $|V|[2,8,20,28,31]$. One of the main interests of this result is that, while $W^{2}$ is chosen so as to behave like a good short-range potential, $V=(\nabla \cdot W)$ can be wildly oscillating either locally or at infinity (for example $V=\frac{1}{r\left(1+r^{2}\right)^{1+\varepsilon}} e^{1 / r}$ $\left.\cdot \cos e^{1 / r}, \varepsilon>0\right)$ in such a way that important cancellations occur between its positive and negative parts, so that asymptotic completeness between $H_{0}+V$ and $H_{0}$ holds. On the contrary, in the example found by Pearson [21] of a very

* Laboratoire associé au Centre National de la Recherche Scientifique 
singular potential violating asymptotic completeness, the positive and negative parts of the potential do not compensate.

As an operator acting on $L^{2}\left(\mathbb{R}^{n}\right) \equiv \mathscr{H}, V$ takes the form $\nabla \cdot W-W \cdot \nabla$, so that $H$ can be written:

$$
H=(-\nabla-W) \cdot(\nabla-W)-W^{2}=-e^{-U} \nabla \cdot e^{2 U} \nabla e^{-U}-W^{2}
$$

if $W$ is the gradient of some $\mathbb{R}$-valued function $U . H$ is therefore a special form of the general case

$$
H=e^{-U}\left(-\nabla \cdot e^{2 U} \nabla+\tilde{V}\right) e^{-U}=e^{-U} H_{1} e^{-U} .
$$

But if $U$ is bounded, $e^{-U}$ is a bijection in $\mathscr{H}$, so that $H$ "resembles" $H_{1}$ in a sense to be made precise; furthermore if $U(x)$ tends suitably to zero when $|x|$ tends to infinity, we expect $-\nabla \cdot e^{2 U} \nabla$ to behave for large $|x|$ very much like the free hamiltonian $H_{0}=-\nabla^{2}$; it will then be enough to make on $\tilde{V}$ the usual assumptions imposed on the potentials. This has been recently pointed out and studied by Pearson [23]. He assumes $V=V_{1}+V_{2}$ is a radial potential such that:

$$
\begin{aligned}
& V_{2}=\frac{d W}{d r} \quad(r=|x|) \\
& W=\frac{d U}{d r} .
\end{aligned}
$$

$H$ can therefore be written in the form (1.1) with:

$$
\tilde{V}=\left(V_{1}-W^{2}\right) e^{2 U} .
$$

Pearson studies the asymptotic behaviour of positive energy generalized eigenfunctions of $H$, either near $r=0$ or near $r=\infty$, and then relies on results on differential equations $[10,13]$ to conclude in favour of asymptotic completeness under the following assumptions:

$(\alpha)$ either there exists $\varepsilon>0$ such that

$$
\begin{aligned}
& U=O\left(r^{-1-\varepsilon}\right) \\
& Q=O\left(r^{-1-\varepsilon}\right)
\end{aligned} \text { as } r \rightarrow \infty
$$

and similar estimates for the convergence at infinity of the integrals defining their Fourier transforms, where $Q$ satisfies

$$
\frac{d Q}{d r}=e^{2 U} \tilde{V}
$$

( $\beta)$ or there exists $\beta: \frac{3}{4}<\beta \leqq 1$ such that:

$$
U=O\left(r^{-\beta}\right) \text { as } r \rightarrow \infty
$$

and some additional conditions on the convergence at infinity of the integrals defining the Fourier transforms of $U, U^{2}, \tilde{V}$. 
This allows him to find a wide class of oscillating potentials leading to asymptotic completeness, among them:

$$
\begin{aligned}
& V=\frac{\sin r}{r}, \\
& V=\frac{\sin r^{\alpha}}{r^{\beta}} \text { with } \beta+2(\alpha-1)>1 \text { and } \frac{3}{2}<\beta+\alpha<2, \\
& V=\frac{1}{2}+e^{r} \cos e^{r}
\end{aligned}
$$

and stranger and wilder ones like:

$$
\begin{aligned}
V= & \frac{1}{2} e^{2(1-\varepsilon) r}+e^{(2-\varepsilon) r} \sin e^{r}+\frac{1}{2} e^{2(1-\varepsilon) r} \cos 2 e^{r} \\
& -(1-\varepsilon) e^{(1-\varepsilon) r} \cos e^{r}, \quad \varepsilon>0
\end{aligned}
$$

(for similar results in situations of the type (1.2) and (1.3) see also [11] and [33]).

In this paper, we extend the result $(\alpha)$ of Pearson to the $n$-dimensional case, recovering potentials (1.3) to (1.5) as particular examples, but not (1.2) which relies on result $(\beta)$ of Pearson. We consider hamiltonians $H$ of the form (1.1) with the following assumptions :

(a) $U \in L^{\infty}\left(\mathbb{R}^{n}\right)$,

(b) the local singularities of $\tilde{V}$ are controlled in a suitable sense by the kinetic energy $H_{0}$,

(c) there exists a $\mathbb{R}^{n}$-valued function of $x$ denoted $Q$, and a $\mathbb{R}$-valued function of $x$ denoted $F$, so that

$$
\tilde{V}=(\nabla \cdot Q)+F,
$$

where $Q$ and $F$ both tend to zero at infinity suitably.

(d) $U, Q$, and $F$ decrease faster than $r^{-1}$ at infinity.

The results are as follows:

(1) $H$ can be defined as a self-adjoint operator in $L^{2}\left(\mathbb{R}^{n}\right)$.

(2) The essential spectrum $\sigma_{e}(H)$ of $H$ is the positive real axis.

(3) The (negative) discrete spectrum of $H$ is finite.

(4) The singular continuous spectrum of $H$ is empty, and the positive point spectrum of $H$ (if any) has 0 as the only possible limit point.

(5) The wave operators $\Omega_{ \pm}$exist as strong limits:

$$
\Omega_{ \pm}=\underset{t \rightarrow \pm \infty}{S-\lim _{t \rightarrow \infty}} e^{i t H} e^{-i t H_{0}}
$$

and are asymptotically complete.

All these properties are derived by standard Hilbert space methods. In particular, under assumptions (a), (b), and (c) only, we define the hamiltonian by a method of quadratic forms, we derive (2), and we use the method of Birman [4] and Schwinger [29] to prove (3). We then assume conditions (a)-(d) to hold, and use the method of Agmon [1] based on a priori estimates in weighted Hilbert spaces to prove (4), and the method of Kato [14] and Lavine [18] of smooth operators to prove (5). As an alternative route, we slightly modify Enss' timedependent method [12] on the more abstract form given by Simon [30] to recover 
results (2), (4), and (5). In this last point, the results and proofs have some analogy to a recent study by Cotta-Ramusino, Krüger and Schrader [7] of quantum scattering on a Riemannian space with asymptotically flat metrics, and YangMills potentials vanishing at infinity.

The paper is organized as follows: Section 2 contains results (1), (2), and (3), together with the definition of auxiliary operators occurring in what follows. Section 3 contains a proof of (4) based on Agmon's estimates of resolvents, and Sect. 4 contains a proof of (5) using the results of Sect. 3 and the method of smooth operators. In Sect. 5 we give an alternative proof of (4), (5), and (2) by Enss' timedependent method.

\section{The Hamiltonian: Its Essential and Discrete Spectra}

Let $\mathscr{H}=L^{2}\left(\mathbb{R}^{n}\right)$, and let $\overline{\mathscr{H}}=\mathscr{H} \otimes \mathbb{C}^{n}$ be the space of square integrable functions from $\mathbb{R}^{n}$ to $\mathbb{C}^{n}$. Let $\|\cdot\|$ and $\langle\cdot, \cdot\rangle$ denote the norm and the scalar product in both of them. We denote by $\nabla$ the operator $\varphi \rightarrow \partial_{j} \varphi$ from $\mathscr{H}$ to $\overline{\mathscr{H}}$. Let $U$ be a real locally integrable function on $\mathbb{R}^{n}$, and let $W$ be its gradient:

$$
W=(\nabla U) \text {. }
$$

Then multiplication by $W$ defines an operator from $\mathscr{H}$ to $\overline{\mathscr{H}}$, also denoted $W$. Similarly, let $Q$ be a real locally integrable function from $\mathbb{R}^{n}$ to $\mathbb{R}^{n}$, and let $(\nabla \cdot Q)$ denote multiplication by the real function $\sum_{j=1}^{n} \partial_{j} Q_{j}$. We define the real multiplicative operator $\tilde{V}$ by:

$$
\tilde{V}=(\nabla \cdot Q)+F
$$

where $F$ is a measurable function from $\mathbb{R}$ to $\mathbb{R}$. We choose $H_{0}=-\Delta$, and we want to define the hamiltonian $H$ which is formally:

$$
\begin{aligned}
H & =-\Delta+(\Delta U)+W^{2}+\tilde{V} e^{-2 U} \\
& =e^{-U}\left(-\nabla \cdot e^{2 U} \nabla+\tilde{V}\right) e^{-U}
\end{aligned}
$$

In all that follows we shall make the following assumptions on the local behaviour of $U, Q$, and $F$ :

$$
U \in L^{\infty}\left(\mathbb{R}^{n}\right) \quad[\text { more precisely }-\infty<\mu \leqq U(x) \leqq v<+\infty] .
$$

For any $a>0$ there exists $b$ and $b^{\prime}$ finite such that:

$$
\begin{aligned}
& Q^{2} \leqq a^{2}\left(H_{0}+b\right), \\
& |F| \leqq a\left(H_{0}+b^{\prime}\right) .
\end{aligned}
$$

(Restrictions on the behaviour at infinity of $U, F$, and $Q$ will be made when necessary.) 
Remark 2.1. Sufficient conditions on $Q$ and $F$ for (2.4) and (2.5) to hold are: $Q^{2}$ and $F \in L_{\text {loc }}^{p}\left(\mathbb{R}^{n}\right)$ with uniform bound, i.e.

$$
\begin{gathered}
\int_{|x-y| \leqq 1} Q^{2}(y)^{p} d^{n} y \equiv q(x) \leqq M, \\
\int_{|x-y| \leqq 1}|F(y)|^{p} d^{n} y \equiv r(x) \leqq M
\end{gathered}
$$

with $M$ not depending on $x$ and with

$$
\left.\begin{array}{lll}
p=1 & \text { for } & n=1 \\
p>1 & \text { for } & n=2 \\
p=\frac{n}{2} & \text { for } & n \geqq 3 .
\end{array}\right\}
$$

For $n=3$, this condition can be weakened to a uniform local Rollnik condition (see [8]).

The case of oscillating potentials studied in [8] is the particular case $\tilde{V}=-W^{2} e^{2 U}=F, Q=0$. In that case the local condition (2.5) is therefore a condition on the local behaviour of $W$. On the contrary, in the general case studied here, no assumption is made on $W$ itself (except that it is the gradient of a bounded function). In particular $U$ may contain a strongly oscillating factor, so that strong local singularities may appear in $W^{2}$ that are not $H_{0}$-bounded, for example: $W=\frac{x}{r^{3}} e^{1 / r} \sin e^{1 / r}(r=|x|)$.

Since $|U|$ is assumed to be bounded, multiplication by the functions $e^{U}$ and $e^{-U}$ define positive self-adjoint operators in $\mathscr{H}$. [We recall that there is a one-to-one correspondence between positive self-adjoint operators and closed positive quadratic forms, such that the domain $Q(A)$ of the closed form associated with the operator $A$ is $\mathscr{D}(\sqrt{A})$ ([16], Chap. VI). We shall in general use the same notation for the operator and the associated quadratic form.] From this and from the assumptions (2.3) to (2.5) we can set the following definition:

Definition 2.1. $H_{1}$ is the semi-bounded self-adjoint operator associated to the closed semi-bounded quadratic form:

$$
-\nabla \cdot e^{2 U} \nabla+\nabla \cdot Q-Q \cdot \nabla+F
$$

defined on $\mathscr{D}(P)$ where $P$ is the self-adjoint operator $-i \nabla$.

Remark 2.2. In order to check that (2.9) is a semi-bounded form it is enough to note that, if $H_{0}=P^{2}$

$$
-\nabla \cdot e^{2 U} \nabla \geqq e^{2 \mu} H_{0}
$$

because of (2.3), and for any $\varphi \in \mathscr{D}(P)$ :

$$
\begin{aligned}
|\langle\varphi,(\nabla \cdot Q-Q \cdot \nabla) \varphi\rangle| & \leqq 2\|\nabla \varphi\|\|Q \varphi\| \\
& \leqq 2\left\{\left\langle\varphi, H_{0} \varphi\right\rangle\left\langle\varphi, a^{2}\left(H_{0}+b\right) \varphi\right\rangle\right\}^{1 / 2} \\
& \leqq a\left\langle\varphi,\left(2 H_{0}+b\right) \varphi\right\rangle
\end{aligned}
$$


so that $\nabla \cdot Q-Q \cdot \nabla+F$ is small with respect to $-\nabla \cdot e^{2 U} \nabla$ in the sense of quadratic forms.

We are then naturally led to the following definition:

Definition 2.2. $H$ and $\tilde{H}_{0}$ are the semi-bounded self-adjoint operators associated respectively with the following semi-bounded closed quadratic forms:

$$
\begin{aligned}
H & =e^{-U} H_{1} e^{-U}, \\
\tilde{H}_{0} & =e^{-U} H_{0} e^{-U}
\end{aligned}
$$

defined on

$$
Q(H)=e^{U} \mathscr{D}(P) \equiv\left\{\varphi: e^{-U} \varphi \in \mathscr{D}(P)\right\},
$$

and let

$$
H_{0}^{\prime}=-\nabla \cdot e^{2 U} \nabla
$$

Remark 2.3. $\tilde{H}_{0}$ will be used in Sect. 4, as an intermediate operator between $H$ and $H_{0}$.

Once $H$ is defined as a self-adjoint operator, a very convenient tool for studying its spectral and scattering properties is its resolvent $R(\lambda)=(H-\lambda)^{-1}$, defined as a bounded operator at least for $\lambda \in \mathbb{C} \backslash \mathbb{R}$. But:

$$
H-\lambda=e^{-U}\left(H_{1}-\lambda e^{2 U}\right) e^{-U}
$$

so that studying the family of operators

$$
H_{1}(\lambda)=H_{1}-\lambda e^{2 U}
$$

will give information on $R(\lambda)$.

Lemma 2.1. (i) Let $\lambda \in \mathbb{C}$ and assume (2.3) to (2.5) hold. Then $H_{1}(\lambda)$ defined by (2.15) is a $m$-sectorial operator with form domain $\mathscr{D}(P)$.

(ii) If $\lambda \in \mathbb{C} \backslash \mathbb{R}, H_{1}(\lambda)$ is invertible, and

$$
\left\|H_{1}(\lambda)^{-1}\right\| \leqq|\operatorname{Im} \lambda|^{-1} e^{-2 \mu} \text {. }
$$

Proof. (i) As $e^{2 U}$ is bounded, this follows from the general result on perturbation of forms (see [16], Theorem 3.4, p. 338).

(ii) From (2.14), we have

$$
\mathscr{R}\left(H_{1}(\lambda)\right)=e^{U} \mathscr{R}(H-\lambda)=\mathscr{H} \quad \text { if } \quad \operatorname{Im} \lambda \neq 0
$$

because $H$ is self-adjoint and $e^{U}$ is bijective. Furthermore:

$$
\|\varphi\|\left\|H_{1}(\lambda) \varphi\right\| \geqq\left|\left\langle\varphi, H_{1}(\lambda) \varphi\right\rangle\right| \geqq e^{2 \mu}|\operatorname{Im} \lambda|\|\varphi\|^{2} .
$$

This implies that $H_{1}(\lambda)$ is invertible, with $\left\|H_{1}(\lambda)^{-1}\right\| \leqq|\operatorname{Im} \lambda|^{-1} e^{-2 \mu}$.

We now turn to the study of the essential spectrum of $H, \sigma_{e}(H)$. Under very weak decrease conditions on $Q$ and $F$ (but not on $U$ !), we prove that $\sigma_{e}(H)$ is what we expect it to be for ordinary potentials: 
Proposition 2.1. Let $U$ satisfy condition (2.3) and let in addition $Q$ and $F$ be such that:

$Q R_{0}(\lambda) Q$ and $|F|^{1 / 2} R_{0}(\lambda)|F|^{1 / 2}$ are compact operators

(respectively in $\overline{\mathscr{H}}$ and in $\mathscr{H}$ ) for some $\lambda<0$.

Then $\sigma_{e}(H) \subset[0, \infty)$.

Remark 2.4. One can give sufficient conditions on $Q$ and $F$ that ensure compactness of $Q R_{0}(\lambda) Q$ and $|F|^{1 / 2} R_{0}(\lambda)|F|^{1 / 2}$ for $\lambda<0$. For instance it is sufficient that $Q^{2}$ and $F$ belong to $L_{\text {loc }}^{P}\left(\mathbb{R}^{n}\right)$ with the same $p$ as in Remark 2.1 and where in addition the $q(x)$ and $r(x)$ defined in (2.6) and (2.7) tend to zero as $x$ tends to infinity. It is clear that the assumptions of Proposition 2.1 are stronger than conditions (2.4) and (2.5).

Proof of Proposition 2.1. It immediately follows from the relation (2.10) between $H$ and $H_{1}$ and from the following two lemmas:

Lemma 2.2. Let $H_{1}$ be as in Definition 2.1 and let $Q$ and $F$ satisfy the assumptions of Proposition 2.1. Then

$$
\sigma_{e}\left(H_{1}\right)=\sigma_{e}\left(H_{0}^{\prime}\right) \subset[0, \infty) .
$$

Proof. It follows from the assumptions that $\nabla \cdot Q-Q \cdot \nabla+F$ is a relatively form compact perturbation of $H_{0}$ and therefore of $H_{0}^{\prime}$. Then from [27, p. 116]

$$
\sigma_{e}\left(H_{1}\right)=\sigma_{e}\left(H_{0}^{\prime}\right)
$$

But

$$
\sigma_{e}\left(H_{0}^{\prime}\right) \subset \sigma\left(H_{0}^{\prime}\right) \subset[0, \infty) .
$$

This completes the proof of Lemma 2.2

Lemma 2.3. Let $H^{\prime}$ be a self-adjoint semi-bounded operator, and let $A$ be a bounded with bounded inverse operator. Then if $H^{\prime \prime}$ is the self-adjoint operator $A^{*} H^{\prime} A$ with domain $A^{-1} \mathscr{D}\left(H^{\prime}\right), \sigma_{e}\left(H^{\prime \prime}\right)$ is contained in $[0, \infty)$ if and only if $\sigma_{e}\left(H^{\prime}\right)$ is.

Proof. We only prove

$$
\sigma_{e}\left(H^{\prime}\right) \subset[0, \infty) \Rightarrow \sigma_{e}\left(H^{\prime \prime}\right) \subset[0, \infty)
$$

because the converse is shown similarly, due to the symmetry between $H^{\prime}=A^{*-1} H^{\prime \prime} A^{-1}$ and $H^{\prime \prime}$. Let $H_{-}^{\prime}$ (resp. $H_{+}^{\prime}$ ) be the negative (resp. positive) part of $H^{\prime}$. Now since $H^{\prime}$ is semi-bounded $H_{-}^{\prime}$ is a bounded operator; furthermore, it is compact if and only if $\sigma_{e}\left(H^{\prime}\right) \subset[0, \infty)$. Let

$$
H_{A}^{ \pm}=A^{*} H_{ \pm}^{\prime} A \text {. }
$$

Then if $\sigma_{e}\left(H^{\prime}\right) \subset[0, \infty), H_{-}^{\prime}$ and therefore $H_{A}^{-}$is compact. This implies

$$
\sigma_{e}\left(H^{\prime \prime}\right)=\sigma_{e}\left(H_{A}^{+}\right) \subset \sigma\left(H_{A}^{+}\right) \subset[0, \infty)
$$

This completes the proof of Lemma 2.3. 
We now turn to the study of the negative spectrum of $H$. From Proposition 2.1 we already know that it is discrete. We prove that if in addition $Q^{2}$ and $F$ tend to zero at infinity faster than $r^{-2}$, then the discrete spectrum of $H$ is finite.

Let $R_{0}(\lambda)$ denote the resolvent operator $\left(H_{0}-\lambda\right)^{-1}$.

Proposition 2.2. Assume $U$ satisfies (2.3) and assume in addition that

$$
Q R_{0}(0) Q \text { and }\left|F_{-}\right|^{1 / 2} R_{0}(0)\left|F_{-}\right|^{1 / 2} \text { are compact, }
$$

where $F_{-}=\min (F, 0)$. Then the number of non-positive eigenvalues of $H$ is finite.

Remark 2.4. For $n \geqq 3$ one can easily give sufficient conditions on $F$ and $Q$ that imply assumption (2.17). For instance it is sufficient that $F$ and $Q^{2}$ belong to $L^{n / 2}\left(\mathbb{R}^{n}\right)$. For $n=3$ this can be weakened to the condition that $Q^{2}$ and $F$ belong to the Rollnik class (see [8]).

For $n=1$, or $n=2,(2.17)$ cannot hold even for smooth $Q$ and $F$ with compact support. However, the argument of Proposition 2.2 can be slightly modified, and the result recovered in these cases, under the following assumptions:

$$
n=1, Q^{2} \text { and } F \in L_{\text {loc }}^{1}(\mathbb{R}),
$$

and

$$
\begin{aligned}
& \lim _{r \rightarrow \infty} r \int_{|x| \geqq r} d x\left(Q^{2}+\left|F_{-}\right|\right)=0, \\
& n=2, Q^{2} \text { and } F \in L_{\mathrm{loc}}^{p}\left(\mathbb{R}^{2}\right), \text { with } p>1,
\end{aligned}
$$

and

$$
Q^{2} \quad \text { and } \quad F=o(|x| \log |x|)^{-2} \quad \text { as } \quad|x| \rightarrow \infty \quad(\text { see }[5]) .
$$

Proof of Proposition 2.2. The proof is an easy extension to the present situation of the well-known argument of Birman [4] and Schwinger [29] which proves the finiteness of the discrete spectrum for ordinary potentials that decrease faster than $r^{-2}$ at infinity (see for example [27, Chap. XIII.3] for details). The result is an immediate consequence of the following two lemmas: [for each self-adjoint operator $K$, we denote by $P_{(-\infty, 0)}(K)$ the spectral projector of $K$ on the interval $(-\infty, 0)]$.

Lemma 2.4. Let $H_{1}$ be as in Definition 2.1 and let $Q$ and $F$ satisfy (2.17). Then $\operatorname{dim} P_{(-\infty, 0]}\left(H^{\prime}\right)$ is finite.

Proof. From (2.3) and (2.9) we have

$$
H_{1} \geqq e^{2 \mu} H_{0}+\nabla \cdot Q-Q \cdot \nabla+F_{-}
$$

which implies

$$
\operatorname{dim} P_{(-\infty, 0)}\left(H_{1}\right) \leqq \operatorname{dim} P_{(-\infty, 0)}\left(H_{0}+e^{-2 \mu}\left(\nabla \cdot Q-Q \cdot \nabla+F_{-}\right)\right) .
$$


But a very simple extension of Schwinger proof (see for example the proof of Proposition 3.2 in [8]) shows that due to (2.17) the R.H.S. of (2.18) is finite. The inclusion of the eigenvalue zero is standard. This completes the proof of Lemma 2.4 .

Lemma 2.5. Let $H^{\prime}, A$ and $H^{\prime \prime}$ be as in Lemma 2.3. Then

$$
\operatorname{dim} P_{(-\infty, 0]}\left(H^{\prime}\right)=\operatorname{dim} P_{(-\infty, 0]}\left(H^{\prime \prime}\right) .
$$

Proof. Due to the symmetry between $H^{\prime}$ and $H^{\prime \prime}$ we only prove

$$
\begin{aligned}
& \operatorname{dim} P_{(-\infty, 0)}\left(H^{\prime \prime}\right) \leqq \operatorname{dim} P_{(-\infty, 0)}\left(H^{\prime}\right), \\
& \operatorname{dim} P_{(-\infty, 0)}\left(H^{\prime}\right)=\operatorname{rank}\left(H_{-}^{\prime}\right)=\operatorname{rank}\left(A^{*} H_{-}^{\prime} A\right)=\operatorname{dim} P_{(-\infty, 0)}\left(H_{A}^{-}\right) .
\end{aligned}
$$

But as

$$
\begin{aligned}
& H^{\prime \prime}=H_{A}^{+}+H_{A}^{-} \geqq H_{A}^{-}, \\
& \operatorname{dim} P_{(-\infty, 0)}\left(H^{\prime \prime}\right) \leqq \operatorname{dim} P_{(-\infty, 0)}\left(H_{A}^{-}\right)=\operatorname{dim} P_{(-\infty, 0)}\left(H^{\prime}\right) .
\end{aligned}
$$

Furthermore one checks easily that the zero eigenvalues of $H^{\prime}$ and $H^{\prime \prime}$ are in oneto-one correspondence, which completes the proof of Lemma 2.5.

\section{The Positive Spectrum of $H$}

In this section, we shall prove that the singular continuous spectrum of $H$ is empty, and that positive energy eigenvalues (when they occur) can only accumulate at zero. We use Agmon's method of a priori estimates in weighted Hilbert spaces, and this will provide us an estimate of the resolvent on the positive real axis, which will be useful for proving asymptotic completeness in the next section. Since this method applies to the present situation with very few modifications, the exposition will be sketchy and some of the proofs omitted; we refer the reader to [27], Chap. XIII.8 for details.

We first introduce some notation:

$$
\mathscr{H}_{\alpha}^{\beta}=\left\{\varphi:\|\varphi\|_{\alpha, \beta}=\left\|\left(1+\mathrm{H}_{0}\right)^{\beta / 2}\left(1+x^{2}\right)^{\alpha / 2} \varphi\right\|<\infty\right\},
$$

where $\|\cdot\|$ denotes the norm in $\mathscr{H}=L^{2}\left(\mathbb{R}^{n}\right)$.

We recall that $\mathscr{B}(\mathscr{K})$ denotes the set of bounded operators in $\mathscr{K}$. The results of this section are as follows:

Proposition 3. Let $\delta>\frac{1}{2}$ and suppose that:

$$
\begin{aligned}
& U\left(1+x^{2}\right)^{\delta} \in L^{\infty}\left(\mathbb{R}^{n}\right), \\
& Q\left(1+x^{2}\right)^{\delta} R_{0}(-1)\left(1+x^{2}\right)^{\delta} Q \in \mathscr{B}(\overline{\mathscr{H}}), \\
& |F|^{1 / 2}\left(1+x^{2}\right)^{\delta / 2} R_{0}(-1)\left(1+x^{2}\right)^{\delta / 2}|F|^{1 / 2} \in \mathscr{B}(\mathscr{H}) .
\end{aligned}
$$

Then:

(1) The positive point spectrum of $H$ is a discrete subset $\mathscr{E}$ of $(0, \infty)$ (with possibly 0 as an accumulation point). Each eigenvalue has finite multiplicity. 
(2) The continuous singular spectrum is empty.

(3) For any compact interval $[a, b] \subset(0, \infty) \backslash \mathscr{E}$, the operator $e^{-U} R(\lambda) e^{-U}$ is a bounded operator from $\mathscr{H}_{\delta}^{-1}$ to $\mathscr{H}_{-\delta}^{1}$ with norm uniformly bounded in $\lambda$ for $a \leqq \operatorname{Re} \lambda \leqq b,|\operatorname{Im} \lambda| \leqq 1$.

Remark 3.1. Intuitively, $U, Q$, and $F$ have to decrease faster than $|x|^{-1}$ at infinity. From Remark 2.1 one easily obtains sufficient conditions on $Q$ and $F$ to imply (3.3) and (3.4). For instance it is enough to take:

$$
Q^{2}\left(1+x^{2}\right)^{2 \delta} \text { and }|F|\left(1+x^{2}\right)^{\delta} \in L_{\mathrm{loc}}^{p}\left(\mathbb{R}^{n}\right)
$$

with uniform bound, and with the same value of $p$ as in Remark 2.1.

The main tool in the proof is a control of $R(\lambda)$ for real positive $\lambda$. As already announced in the previous section, we shall first study the family $H_{1}(\lambda)$ defined in (2.15). Lemma 2.1 has already shown that, away from the real axis, its inverse exists and looks like a resolvent. We now show that in fact, $H_{1}(\lambda)^{-1}$ obeys some sort of resolvent identity that allows us to approach the positive real axis under suitable decrease properties of $U, Q$, and $F$.

$$
\begin{aligned}
H_{1}(\lambda) & =H_{0}-\lambda-\nabla \cdot\left(e^{2 U}-1\right) \nabla+[\nabla, Q]+F-\lambda\left(e^{2 U}-1\right) \\
& =H_{0}-\lambda+\sum_{j=1}^{5} A_{j}^{*} B_{j}
\end{aligned}
$$

with

$$
\left.\begin{array}{ll}
A_{1}=\left(e^{2 U}-1\right)^{1 / 2} \nabla & B_{1}=\left|e^{2 U}-1\right|^{1 / 2} \nabla \\
A_{2}=-\left(1+x^{2}\right)^{-\delta / 2} \nabla & B_{2}=\left(1+x^{2}\right)^{\delta / 2} Q \\
A_{3}=B_{2} & B_{3}=A_{2} \\
A_{4}=F^{1 / 2} & B_{4}=|F|^{1 / 2} \\
A_{5}=-\lambda^{1 / 2}\left(e^{2 U}-1\right)^{1 / 2} & B_{5}=\lambda^{1 / 2}\left|e^{2 U}-1\right|^{1 / 2},
\end{array}\right\}
$$

where $f^{1 / 2}=\frac{f}{|f|^{1 / 2}}$.

The $A_{j}$ 's and $B_{j}$ 's are operators from $\mathscr{H}$ to $\overline{\mathscr{H}}$ or $\mathscr{H}$. For any non real $\lambda$ in a compact $K$ of $\mathbb{C},(3.5)$ implies :

$$
H_{1}(\lambda)^{-1}=R_{0}(\lambda)-R_{0}(\lambda) \sum_{j=1}^{5} A_{j}^{*} B_{j} H_{1}(\lambda)^{-1}
$$

Thanks to the factorization in $A_{j}^{*} B_{j}$, we are in a position to proceed as in the proof of [8, Proposition 4.1] by writing (3.7) as an integral equation with a symmetrized kernel, hopefully compact, in $\hat{\mathscr{H}}=\overline{\mathscr{H}} \oplus \overline{\mathscr{H}} \oplus \overline{\mathscr{H}} \oplus \mathscr{H} \oplus \mathscr{H}$, whose elements are $B_{i} R_{0}(\lambda) A_{j}^{*}$. But we easily see that if $i=j=1$, this element is not compact because it lacks a negative power in $P=-i \nabla$. We overcome this minor difficulty by writing:

$$
H_{1}(\lambda)^{-1}=\left(H_{1}(\lambda)+z\right)^{-1}+z\left(H_{1}(\lambda)+z\right)^{-1} H_{1}(\lambda)^{-1},
$$

where $-z$ is chosen sufficiently negative to be in the resolvent set of all $m$-sectorial operators $H_{1}(\lambda)$ for $\lambda \in K$. We then insert (3.8) in (3.7), and we want to prove that, 
up to an exceptional set $\mathscr{E}$ of values of $\lambda$, (3.7) is equivalent to:

$$
\begin{aligned}
H_{1}(\lambda)^{-1}= & R_{0}(\lambda)-R_{0}(\lambda) \sum_{j=1}^{5} A_{j}^{*} B_{j}\left(H_{1}(\lambda)+z\right)^{-1} \\
& -z R_{0}(\lambda)\left(A_{1}^{*}, \ldots, A_{5}^{*}\right)(\mathbb{1}+F(\lambda))^{-1}\left(\begin{array}{c}
B_{1} \\
\vdots \\
B_{5}
\end{array}\right) \\
& \cdot\left(H_{1}(\lambda)+z\right)^{-1} R_{0}(\lambda)\left(\mathbb{1}-\sum_{j=1}^{5} A_{j}^{*} B_{j}\left(H_{1}(\lambda)+z\right)^{-1}\right),
\end{aligned}
$$

where

$$
F(\lambda) \equiv z\left(\begin{array}{c}
B_{1} \\
\vdots \\
B_{5}
\end{array}\right)\left(H_{1}(\lambda)+z\right)^{-1} R_{0}(\lambda)\left(A_{1}^{*}, \ldots, A_{5}^{*}\right)
$$

is a compact operator in $\hat{\mathscr{H}}$. The key of the proof is the following property:

Lemma 3.1. Let $\delta$ be determined by conditions (3.2) to (3.4) and let $\delta^{\prime}$ be such that $\frac{1}{2}<\delta^{\prime}<\min (\delta, 1)$. Then:

(i) $R_{0}(\lambda)$ is a bounded operator from $\mathscr{H}_{\delta}^{-1}$ to $\mathscr{H}_{-\delta^{\prime}}^{1}$ uniformly for $\lambda \in K$ ( $K$ is any compact of $\mathbb{C} \backslash\{0\})$ and is norm continuous in $\lambda$ for $\lambda \neq 0$.

(ii) $A_{j}$ are bounded operators from $\mathscr{H}_{-\delta}^{1}$ to $\overline{\mathscr{H}}$.

(iii) $B_{j}$ are bounded operators from $\mathscr{H}_{-\delta}^{1}$ to $\overline{\mathscr{H}}$.

(iv) Let $-z$ real belong to the resolvent set of all $H_{1}(\lambda)$ for all $\lambda \in K$ ( $K$ compact in $\mathbb{C}$ ). Then $\left(H_{1}(\lambda)+z\right)^{-1}$ is compact from $\mathscr{H}_{-\delta^{\prime}}^{1}$ to $\mathscr{H}_{-\delta}^{1}$ and is continuous for $\lambda \in K$.

Proof. (i) is a well-known result (see [27], Sect. XIII). (ii) and (iii) easily follow from (3.3) and (3.4) (see for example [8], Lemma 4.2 for the proof of a similar result).

We now prove (iv). It is enough to check that the operator

$$
\left(1+H_{0}\right)^{1 / 2}\left(1+x^{2}\right)^{-\delta / 2}\left(H_{1}(\lambda)+z\right)^{-1}\left(1+x^{2}\right)^{\delta^{\prime} / 2}\left(1+H_{0}\right)^{-1 / 2}
$$

is compact in $\mathscr{H}$. But (3.11) can be rewritten as:

$$
\begin{aligned}
(1+ & \left.H_{0}\right)^{1 / 2}\left(H_{1}(\lambda)+z\right)^{-1}\left(1+x^{2}\right)^{-\frac{\delta-\delta^{\prime}}{2}}\left(1+H_{0}\right)^{-1 / 2} \\
& +\left(1+H_{0}\right)^{1 / 2}\left(H_{1}(\lambda)+z\right)^{-1}\left[H_{1},\left(1+x^{2}\right)^{-\delta / 2}\right]\left(H_{1}(\lambda)+z\right)^{-1} \\
& \cdot\left(1+x^{2}\right)^{\delta^{\prime} / 2}\left(1+H_{0}\right)^{-1 / 2} .
\end{aligned}
$$

As $\delta>\delta^{\prime}$, the factor $\left(1+x^{2}\right)^{-(1 / 2)\left(\delta-\delta^{\prime}\right)}\left(1+H_{0}\right)^{-1 / 2}$ in the first term of (3.12) is compact, and the first factor is bounded and continuous by Lemma 3.2 below. The second term of (3.12) can be rewritten as

$$
\begin{aligned}
& \left(H_{0}+1\right)^{1 / 2}\left(H_{1}(\lambda)+z\right)^{-1}\left[H_{1},\left(1+x^{2}\right)^{-\delta / 2}\right]\left(1+x^{2}\right)^{\delta^{\prime} / 2}\left(H_{1}(\lambda)+z\right)^{-1} \\
& \quad \times\left(H_{0}+1\right)^{-1 / 2}-\left(H_{0}+1\right)^{1 / 2}\left(H_{1}(\lambda)+z\right)^{-1}\left[H_{1},\left(1+x^{2}\right)^{-\delta / 2}\right] \\
& \quad \times\left(H_{1}(\lambda)+z\right)^{-1}\left[H_{1},\left(1+x^{2}\right)^{\delta^{\prime} / 2}\right]\left(H_{1}(\lambda)+z\right)^{-1}\left(1+H_{0}\right)^{-1 / 2} .
\end{aligned}
$$

Now since:

$$
\left[H_{1}, f(x)\right]=-\nabla \cdot e^{2 U}(\nabla f)-e^{2 U}(\nabla f) \cdot \nabla
$$


and since $\left(\nabla\left(1+x^{2}\right)^{\delta^{\prime} / 2}\right)$ is a bounded function of $x$ because $\delta^{\prime}<1$, compactness and continuity of (3.13) easily follow from compactness of $\left(1+x^{2}\right)^{-\alpha}\left(1+H_{0}\right)^{-1 / 2}$ if $\alpha>0$, and from Lemma 3.2:

Lemma 3.2. Let $K$ and $z$ be as in Lemma 3.1(iv). Then:

$\left(1+H_{0}\right)^{1 / 2}\left(H_{1}(\lambda)+z\right)^{-1}\left(1+H_{0}\right)^{1 / 2}$ is bounded in $\mathscr{H}$ and is norm continuous with respect to $\lambda$ in $K$.

Proof. This easily follows from Lemma 2.1(i). We omit the details.

Let $\overline{\mathbb{C}}$ denote the closed cut plane, i.e. the complex plane cut along $[0, \infty)$, including the cut counted twice. Lemma 3.1 then immediately implies:

Corollary 3.1. Let $F(\lambda)$ be defined by (3.10) and (3.6). Then for any compact $K$ of $\overline{\mathbb{C}} \backslash\{0\}$ :

(i) $F(\lambda)$ is a bounded operator in $\hat{\mathscr{H}}$ and is norm continuous w.r.t. $\lambda$ for $\lambda \in K$, with norm uniformly bounded for $\lambda \in K$.

(ii) $F(\lambda)$ is analytic w.r.t. $\lambda$ for $\lambda \in \mathbb{C} \backslash[0, \infty)$

(iii) $F(\lambda)$ is compact for all $\lambda \in K$.

We are now in a position to apply the analytic Fredholm theorem ([24], p. 201) to invert the operator $\mathbb{1}+F(\lambda)$ :

Lemma 3.3. Let $\mathscr{E}_{k}$ be the set of positive $\lambda$ in $K$ for which the homogeneous equation:

$$
[\mathbb{1}+F(\lambda)] \Phi=0
$$

has a solution in $\hat{\mathscr{H}}$, and let $\mathscr{E}=\bigcup_{K} \mathscr{E}_{K}$. Then:

(i) $\mathscr{E} \cup\{0\}$ is a bounded closed set of Lebesgue measure zero (general result of Kuroda [17])

(ii) For any compact interval $[a, b] \subset(0, \infty) \backslash \mathscr{E}, H_{1}(\lambda)^{-1}$ is a bounded operator from $\mathscr{H}_{\delta}^{-1}$ to $\mathscr{H}_{-\delta}^{1}$ with norm uniformly bounded in $\lambda$ for $a \leqq \operatorname{Re} \lambda \leqq b,|\operatorname{Im} \lambda| \leqq 1$.

Proof of Proposition 3. From (2.14) and (2.15), we have

$$
e^{-U} R(\lambda) e^{-U}=H_{1}(\lambda)^{-1}
$$

for all $\lambda \in \mathbb{C} \backslash \mathbb{R}$ where both members are analytic. Therefore Lemma 3.3 (ii) is Proposition 3 (3). For the proof of Proposition 3 (1) and 3 (2), one first shows that solutions of (3.15) satisfy a suitable vanishing property on the energy shell. (We denote by $\mathscr{F}$ the Fourier transform and $k$ the momentum variable.)

Lemma 3.4. Let $\Phi=\left(\Phi_{1}, \ldots, \Phi_{5}\right) \in \hat{\mathscr{H}}$ be a solution of (3.15) with $\lambda>0$. Then:

$$
\left.\mathscr{F}\left(\sum_{j=1}^{5} A_{j}^{*} \Phi_{j}\right)\right|_{k^{2}=\lambda}=0 .
$$

Proof. Let $\lambda>0$ be in $\mathscr{E}$. We suppose that $z$ is chosen sufficiently large in order that

$$
\left\|\left(\begin{array}{c}
B_{1} \\
\vdots \\
B_{5}
\end{array}\right)\left(H_{1}(\lambda)+z\right)^{-1}\left(A_{1}^{*}, \ldots, A_{5}^{*}\right)\right\|<1
$$


as an operator norm in $\hat{\mathscr{H}}$. As $F(\lambda)$ is continuous with respect to $\lambda$ in $\overline{\mathbb{C}} \backslash\{0\}$, we have:

$$
-\Phi=F(\lambda) \Phi=\lim _{\varepsilon \downarrow 0} F(\lambda+i \varepsilon) \Phi .
$$

Then if we replace $z\left(H_{1}(\lambda)+z\right)^{-1}$ by

$$
1-\left(H_{1}(\lambda)+z\right)^{-1}\left(H_{0}-\lambda+\sum_{j=1}^{5} A_{j}^{*} B_{j}\right)
$$

in the definition of $F(\lambda)$, we get:

$$
\begin{gathered}
\lim _{i \neq 0}\left(\mathbb{1}-\left(\begin{array}{c}
B_{1} \\
\dot{B}_{5}
\end{array}\right)\left(H_{1}(\lambda+i \varepsilon)+z\right)^{-1}\left(A_{1}^{*}, \ldots, A_{5}^{*}\right)\right) \\
\cdot\left(\mathbb{1}+\left(\begin{array}{c}
B_{1} \\
\vdots \\
B_{5}
\end{array}\right) R_{0}(\lambda+i \varepsilon)\left(A_{1}^{*}, \ldots, A_{5}^{*}\right)\right) \Phi=0 .
\end{gathered}
$$

But, from (3.17), the operator in the first factor of (3.18) has a limit as $\varepsilon \downarrow 0$ which is invertible, and therefore:

$$
\Phi=-\lim _{\varepsilon \downarrow 0}\left(\begin{array}{c}
B_{1} \\
\vdots \\
B_{5}
\end{array}\right) R_{0}(\lambda+i \varepsilon)\left(A_{1}^{*}, \ldots, A_{5}^{*}\right) \Phi
$$

(where $\varepsilon$ is set equal to zero in $A_{5}^{*}$ and $B_{5}$ ).

Let $s(f)=\frac{f}{|f|}$ for every real non zero $f$, and $s(f)=0$ if $f=0$.

Then (3.19) implies:

$$
\begin{aligned}
& \left\langle\Phi,\left(\begin{array}{ccccc}
s(U) & 0 & 0 & 0 & 0 \\
0 & 0 & 1 & 0 & 0 \\
0 & 1 & 0 & 0 & 0 \\
0 & 0 & 0 & s(F) & 0 \\
0 & 0 & 0 & 0 & -s(U)
\end{array}\right) \Phi\right\rangle \\
& =\lim _{i, 0}\left\langle\Phi,\left(\begin{array}{c}
A_{1} \\
\vdots \\
A_{5}
\end{array}\right) R_{0}(\lambda+i \varepsilon)\left(A_{1}^{*}, \ldots, A_{5}^{*}\right) \Phi\right\rangle .
\end{aligned}
$$

Therefore taking the imaginary part of both members of (3.20) yields:

$$
0=\lim _{\varepsilon \downarrow 0} \varepsilon\left\|R_{0}(\lambda+i \varepsilon) \sum_{1 \leqq j \leqq 5} A_{j}^{*} \Phi_{j}\right\|^{2} .
$$

Now (3.16) is deduced from (3.21) by the standard argument (see [25], Chap. IX.9).

The end of the proof of Proposition 3 (1) and 3 (2) is almost identical with Agmon's for ordinary potentials and we only sketch it. Once Lemma 3.4 is satisfied, we use Agmon's bootstrap argument (see [25], Theorem IX.41) to prove that:

$$
\Psi=R_{0}(\lambda) \sum_{j=1}^{5} A_{j}^{*} \Phi_{j}
$$


is in some $\mathscr{H}_{\varepsilon}^{0}$ with $\varepsilon>0$, and that:

$$
\|\Psi\|_{\varepsilon, 0} \leqq C\|\Phi\|
$$

uniformly for $\lambda$ satisfying $0<a \leqq \lambda \leqq b$.

But from (3.22) the following relation holds between elements of $\mathscr{H}$ :

$$
\left(H_{0}-\lambda\right) \Psi=-\sum_{j=1}^{5} A_{j}^{*} B_{j} \Psi
$$

which implies by $(2.14)$ :

$$
(H-\lambda) e^{U} \Psi=e^{-U}\left(H_{1}-\lambda e^{2 U}\right) \Psi=0 .
$$

This says that $e^{U} \Psi$ is an eigenvector of $H$ with eigenvalue $\lambda$. (Each point of $\mathscr{E}$ is an eigenvalue of $H$.) Furthermore (3.23) says that the set of eigenvectors of $H$ with eigenvalue $\lambda$ in the interval $[a, b](0<a<b<\infty)$ is compact and therefore finite dimensional. This completes the proof.

\section{Existence and Asymptotic Completeness of the Wave Operators}

In this section we prove the existence and asymptotic completeness of the wave operators (1.6) by using Proposition 3 and the method of smooth operators of Kato [14] and Lavine [18]. The results are as follows:

Proposition 4. Assume conditions (3.2) to (3.4) to hold with $\delta>\frac{1}{2}$. Then the wave operators (1.6) exist and are asymptotically complete.

Proof. We use $\tilde{H}_{0}$ defined by $(2.11)$ as an intermediate hamiltonian between $H_{0}$ and $H$; by the chain rule $[16$, Chap. X] the result follows immediately from Lemmas 4.1 and 4.2 below.

Remark 4.1. We first notice that under assumption (3.2) alone, the analog of Proposition 3 can be shown for $\tilde{H}_{0}$ instead of $H$. Therefore there exists a discrete subset of $(0, \infty)$ denoted $\tilde{\mathscr{E}}$ such that $\tilde{\mathscr{E}} \cup\{0\}$ is closed, $\tilde{H}_{0}$ is absolutely continuous away from $\tilde{\mathscr{E}}$, and $\left(1+x^{2}\right)^{-\delta / 2}$ and

$$
\left(1+x^{2}\right)^{-\delta / 2}\left(H_{0}+1\right)^{1 / 2} e^{-U}
$$

are $\tilde{H}_{0}$-smooth on any interval $[a, b] \subset(0, \infty) \backslash \tilde{\mathscr{E}}$. [We recall that $\delta>\frac{1}{2}$ is the number occurring in assumption (3.2).] Furthermore from theorems on the absence of positive eigenvalues [27, Chap. XIII.13] and from assumption (3.2) one can show that $\tilde{\mathscr{E}}=\emptyset$.

As a first step enabling one to go from $H_{0}$ to $\tilde{H}_{0}$ we have:

Lemma 4.1. Assume $U$ satisfies (3.2) with $\delta>\frac{1}{2}$. Then the wave operators

$$
\Omega_{ \pm}\left(\tilde{H}_{0}, H_{0}\right)=\underset{t \rightarrow \pm \infty}{s-\lim _{t \rightarrow \infty}} e^{i t \tilde{H}_{0}} e^{-i t H_{0}}
$$

exist and are asymptotically complete. 
Proof. As $\left|e^{ \pm U}-1\right| \leqq|U| \operatorname{Max}\left(e^{v}, e^{-\mu}\right)$, it follows from assumption (3.2) that $e^{-U}-1$ is $H_{0}$-compact. It is also $\tilde{H}_{0}$-compact because :

$$
\begin{aligned}
\left(e^{-U}-1\right)\left(\tilde{H}_{0}+i\right)^{-1}= & \left(1-e^{U}\right)\left(H_{0}+i\right)^{-1} e^{U} \\
& -i\left(1-e^{U}\right)\left(H_{0}+i\right)^{-1}\left(e^{U}-e^{-U}\right)\left(\tilde{H}_{0}+i\right)^{-1} .
\end{aligned}
$$

By a standard approximation argument [26] this implies:



and similarly for $\tilde{H}_{0}$. Therefore it is enough to prove the existence of the limits:

$$
\underset{\substack{S-\lim _{t \rightarrow \pm \infty} \\ i t}}{i \tilde{H}_{0}} e^{U} e^{-i t H_{0}}
$$

and

$$
\underset{t \rightarrow \pm \infty}{S-\lim _{t \rightarrow \infty}} e^{i t H_{0}} e^{-U} e^{-i t \tilde{H}_{0}}
$$

(because $\tilde{H}_{0}$ is absolutely continuous by Remark 4.1), and if the limits (4.4) and (4.5) exist, they are equal respectively to $\Omega_{ \pm}\left(\tilde{H}_{0}, H_{0}\right)$ and $\Omega_{ \pm}\left(\tilde{H}_{0}, H_{0}\right)^{*}$. But as sesquilinear forms on $\mathscr{D}\left(\tilde{H}_{0}\right) \times \mathscr{D}\left(H_{0}\right)$ and on $\mathscr{D}\left(H_{0}\right) \times \mathscr{D}\left(\tilde{H}_{0}\right)$ respectively, we have:

$$
\begin{aligned}
\tilde{H}_{0} e^{U}-e^{U} H_{0} & =\left(e^{-U}-e^{U}\right) H_{0}, \\
H_{0} e^{-U}-e^{-U} \tilde{H}_{0} & =\left(e^{U}-e^{-U}\right) \tilde{H}_{0} .
\end{aligned}
$$

Now by assumption (3.2) there exists a constant $C<\infty$ such that:

$$
\left|e^{U}-e^{-U}\right| \leqq C\left(1+x^{2}\right)^{-\delta}, \quad \delta>\frac{1}{2} .
$$

As $\left(1+x^{2}\right)^{-\delta / 2}$ is $H_{0}$ - and $\tilde{H}_{0}$-smooth, this implies the existence of the limits (4.4) and (4.5) on the dense sets $\mathscr{R}\left(E_{H_{0}}(I)\right)$ and $\mathscr{R}\left(E_{\tilde{H}_{0}}(I)\right)$ respectively [where $I$ goes through the set of compact intervals of $\mathbb{R}$ and $E_{G}(I)$ denotes the spectral projector of $G$ on the interval $I]$. This completes the proof.

The second result allows one to go from $\tilde{H}_{0}$ to $H$ :

Lemma 4.2. Let $U, Q$, and $F$ satisfy (3.2), (3.3), and (3.4), and $H$ and $\tilde{H}_{0}$ be defined by Definition 2.2. Then the wave operators:

$$
\Omega_{ \pm}\left(H, \tilde{H}_{0}\right)=\underset{t \rightarrow \pm \infty}{S-\lim _{t \rightarrow \infty}} e^{i t H} e^{-i t \widetilde{H}_{0}}
$$

exist and are asymptotically complete.

Proof. As quadratic forms on $Q(H)=Q\left(\tilde{H}_{0}\right)=e^{U} Q\left(H_{0}\right)$ we have:

$$
\begin{aligned}
H-\tilde{H}_{0} & =-e^{-U} \nabla \cdot\left(e^{2 U}-1\right) \nabla e^{-U}+e^{-U}[\nabla, Q] e^{-U}+F e^{-2 U} \\
& =e^{-U} \sum_{j=1}^{4} A_{j}^{*} B_{j} e^{-U},
\end{aligned}
$$

where $A_{j}$ and $B_{j}$ are defined in (3.6). It is clear that:

$$
\mathscr{D}(H) \subset \mathscr{D}\left(A_{j} e^{-U}\right) \quad \text { and } \quad \mathscr{D}\left(\tilde{H}_{0}\right) \subset \mathscr{D}\left(B_{j} e^{-U}\right), \quad j=1, \ldots, 4 .
$$


Moreover Proposition 3 (3) shows that the expression (4.1) is $H$-smooth on any interval $[a, b] \subset(0, \infty) \backslash \mathscr{E}$ and therefore, so are $A_{j} e^{-U}$ for $j=1, \ldots, 4$. Furthermore it follows from Remark 4.1 that $B_{j} e^{-U}$ are $\tilde{H}_{0}$-smooth on $[a, b]$. But the absolutely continuous spectra of $H$ and $\tilde{H}_{0}$ are exhausted by a denumerable union of such intervals. By application of the $H$-smoothness theory, this implies the result.

\section{Time-Dependent Methods}

In this section, we recover results (2), (4), and (5) of the introduction by an extension to the present situation of Enss' purely time-dependent method [12], in the following directions:

(1) $H$ is defined by means of quadratic forms rather than as an operator perturbation of $H_{0}$.

(2) Here, $H$ and $H_{0}$ are not in general "mutually subordinate" in the sense of Birman [6]. Instead, we have an "identification operator" (see [3] and [15]) $J=e^{U}$ that sends $Q\left(H_{0}\right)$ onto $Q(H)$ and that is close to one when $|x|$ is large.

But these extensions are precisely contained in great part in the abstract form of Enss' result given by Simon [30]; namely, extensions in the directions (1) and (2) are considered respectively in Sects. 4 and 6 of [30] (see also [34]). We stick them together in the following theorem:

Theorem 1. Let $H_{0}=-\Delta$, and $H$ be a self-adjoint operator, such that:

(a) There exists bounded operators $J$ and $J^{\prime}$ with $1-J$ and $1-J^{\prime} H_{0}$-compact.

(b) For any fixed interval $I_{0}$

$\left\|E(\mathbb{R} \backslash I) J E_{0}\left(I_{0}\right)\right\| \rightarrow 0$ when the interval I tends to $\infty$,

where $E(I)$ (resp. $E_{0}\left(I_{0}\right)$ ) is the spectral projector of $H$ (resp. $H_{0}$ ) on the interval $I$ (resp. $I_{0}$ ).

(c) $J^{\prime}$ is invertible, and for any fixed interval $I$

$\left\|E_{0}\left(\mathbb{R} \backslash I_{0}\right) J^{\prime} E(I)\right\| \rightarrow 0 \quad$ when the interval $I_{0}$ tends to $\infty$.

(d) For bounded intervals $I$ and $I_{0}$, let:

$$
h(r)=\left\|E(I)\left(H J-J H_{0}\right) E_{0}\left(I_{0}\right) E(|x| \geqq r)\right\|
$$

be such that:

$h(0)<\infty$ and $\int_{0}^{\infty} d r h(r)<\infty$,

where $E(|x| \geqq r)$ is the characteristic function of the set $\{x:|x| \geqq r\}$.

Then:

(i) The wave operators (1.6) exist.

(ii) $H$ has no continuous singular spectrum.

(iii) $\mathscr{R}\left(\Omega_{+}\right)=\mathscr{R}\left(\Omega_{-}\right)$coincide with the absolutely continuous subspace for $H$ (i.e. $\Omega_{ \pm}$are asymptotically complete). 
(iv) Any strictly positive eigenvalue for $H$ has finite multiplicity, and the only possible limit point for the positive point spectrum of $H$ is 0 .

(v) $\sigma_{e}(H)=\sigma_{e}\left(H_{0}\right)=[0, \infty)$.

Remark 5.1. The general case of a "vaguely elliptic" $H_{0}$ works as well, although we do not write it down for simplicity.

Remark 5.2. As we will show in part ( $\alpha$ ) of the proof of Theorem 1, assumptions (a) and (c) imply that $E(|x| \leqq R) E(I)$ is compact for $I$ a bounded interval in $\mathbb{R}$. This expresses the absence of local absorption for $H$, which is a preliminary to asymptotic completeness (see $[9,22]$ ). In this respect, the invertibility of $J^{\prime}$ plays an essential role: namely if $H=H_{0}+V$ with $V$ the potential of [21] which is highly singular at the origin, and if $J^{\prime}$ is zero in some neighborhood of the origin, and one outside, the condition (5.2) that " $H_{0}$ is subordinate to $H$ through $J$ '" is fulfilled, whereas absorption at the origin occurs, so that asymptotic completeness is violated.

Before giving, for completeness, some hints on the proof of Theorem 1, we give the result of this section which is an application of Theorem 1 to the case under study:

Proposition 5. Let $U, Q$, and $F$ satisfy (2.3), (2.4), and (2.5) and suppose in addition that :

$$
\begin{aligned}
& \int_{0}^{\infty} d r\left\|\left(e^{U}-1\right) E(|x| \geqq r)\right\|<\infty, \\
& \int_{0}^{\infty} d r\left\|Q\left(1+H_{0}\right)^{-1 / 2} E(|x| \geqq r)\right\|<\infty, \\
& \int_{0}^{\infty} d r\left\|\left(1+H_{0}\right)^{-1 / 2} F\left(1+H_{0}\right)^{-1 / 2} E(|x| \geqq r)\right\|<\infty,
\end{aligned}
$$

where $E(|x| \geqq r)$ is as in Theorem 1. Let $H$ be defined by (2.9) and (2.10), and $H_{0}=-\Delta$.

Then all the conclusions of Theorem 1 hold.

Remark 5.3. Conditions (5.4)-(5.6) are weaker than (3.2)-(3.4) for obtaining results (4) and (5) of the introduction (no explicit $|x|^{-\delta}$ decrease at infinity with $\delta>1$ is required, but only integrability at infinity). But on the other hand, conditions (5.4)-(5.6) are much more restrictive than the assumptions of Proposition 2.1 as far as the localization of the essential spectrum is concerned. This is one reason why, all along the previous sections, we have sliced the various spectral and scattering properties of $H$ according to the various properties of $U, Q$, and $F$ they require. Furthermore, the purely time-dependent method of this section does not provide the detailed estimates of the resolvent of $H$ contained in Proposition 3 (3).

Proof of Proposition 5. For $J=e^{U}, J^{\prime}=e^{-U}$, assumption (a) of Theorem 1 follows from (3.2) and (5.4): namely the integrand of (5.4) is monotone decreasing and integrable with respect to $r$, and therefore tends to zero as $r$ tends to infinity, which implies that $(1-J)\left(H_{0}+1\right)^{-\alpha}$ and $\left(1-J^{\prime}\right)\left(H_{0}+1\right)^{-\alpha}(\alpha>0)$ are norm limits of the 
compact operators $E(|x| \leqq r)\left(H_{0}+1\right)^{-\alpha}$. Furthermore, $Q(H)=e^{U} Q\left(H_{0}\right)$ implies (b) and (c) $\left[J^{\prime}=e^{-U}\right.$ is obviously invertible from (2.3)]. Now assumption (d) of Theorem 1 easily follows from (2.4), (2.5) and from (5.4)-(5.6) because of the relation:

$$
\begin{aligned}
H J-J H_{0}= & -e^{-U} \nabla \cdot\left(e^{2 U}-1\right) \nabla+\left(e^{-U}-e^{U}\right) H_{0} \\
& +e^{-U}[\nabla, Q]+F e^{-U} .
\end{aligned}
$$

(We omit the details).

Hints for the Proof of Theorem 1

We only mention the points where Theorem 2.2 of [30] is modified in order to accommodate the assumptions of our Theorem 1 .

$(\alpha)$ Let us first notice that assumptions (a) and (c) imply that $(1-J) E(I)$, $\left(1-J^{\prime}\right) E(I)$, and $E(|x| \leqq r) E(I)$ are compact for $I$ a bounded interval of $\mathbb{R}$.

$(\beta)$ Now it easily follows from assumption $(d)$ that:

$$
E\left(I_{1}\right)\left\{\varphi(H) J-J \varphi\left(H_{0}\right)\right\} E_{0}\left(I_{0}\right)
$$

is compact for $\varphi$ a smooth function, and $I_{1}$ and $I_{0}$ bounded intervals.

$(\gamma)$ But $(\alpha)$ and $(\beta)$ imply that $(5.8)$, with $E_{0}\left(I_{0}\right)$ replaced by $E(I)$, is also compact; which in turn implies that $E\left(I_{1}\right)\left\{\varphi(H)-\varphi\left(H_{0}\right)\right\} E(I)$ is compact from assumption (a) and from $(\alpha)$ again.

This is enough for yielding the decomposition

$$
\Phi_{n}=\Phi_{n, \text { in }}+\Phi_{n, \text { out }}+\Phi_{n, w} \text { with }\left\|\Phi_{n, w}\right\| \rightarrow 0 n \rightarrow \infty,
$$

for $\Phi_{n}$ the sequence of Theorem 2.2 in [30] such that $E(I) \Phi_{n}=\Phi_{n}$ for some $I$, and $\Phi_{n}$ weakly converges to zero.

$(\delta)$ Now it remains to be shown that:

$$
\left\|\left(\Omega_{ \pm}-1\right) \Phi_{n, \text { in }}\right\|
$$

tends to zero as $n$ tends to infinity. But from assumption (a):

$$
\Omega_{ \pm}=\underset{t \rightarrow \pm \infty}{S-\lim _{t}} e^{i t H} J e^{-i t H_{0}}
$$

and 1 can be replaced by $J$ in (5.9). Furthermore from assumption (b) it is enough to control:

$$
E\left(I_{1}\right) \int_{0}^{ \pm \infty} d t e^{i t H}\left(H J-J H_{0}\right) e^{-i t H_{0}} \Phi_{n, \text { in }}{ }_{\text {out }}
$$

for $I_{1}$ an interval of $\mathbb{R}$ sufficiently large. Now the support property in momentumspace of $\Phi_{n, \text { in }}$ makes it possible to use assumption (d) in order to complete the estimation of (5.10) along the line of Theorem 2.2 of [30].

Remark 5.4. As already mentioned in the introduction, Proposition 5 bears some resemblance with what is done in [7], (see also references quoted therein). The authors consider quantum scattering by an external metric $g$ and a Yang-Mills 
potential $A$. The analogy with our Hamiltonian (1.1) is suggested by following correspondence:

$g$ corresponds in a certain sense to our bounded function $e^{U}$,

$A$ would correspond to a purely imaginary vector field $Q e^{2 U}$.

Under the assumptions that $A$ and its first $x$-derivative, and the matrix $g-1$ and its first and second $x$-derivative go to zero at infinity faster than $|x|^{-1-\varepsilon}$, they prove the existence and completeness of the wave operators. Moreover they point out the connection between such hamiltonians and positive energy bound states like the Wigner-von Neuman example [32]. Similarly, as far as Hamiltonians (1.1) are concerned, the examples (1.2) and (1.3) of allowed potentials in [23] suggest the existence of such positive energy eigenvalues (see for example $[19,10]$ ). However, (but this may be only a technical point) an important difference with what we do in this paper is the regularity conditions imposed on $A$ and $g$ in [7]; under these regularity conditions, that do not allow for too wild oscillations, $H$ and $H_{0}$ are mutually subordinate, so that Enss' method applies directly.

Acknowledgements. I am grateful to K. Chadan, J. Ginibre, and D. B. Pearson for numerous illuminating discussions, and especially to D.B.Pearson for communicating his results prior to publication.

I am also indebted to the referee for suggesting some improvements in an earlier form of Sect. 2 of this paper, and for bringing [33] to my attention.

\section{References}

1. Agmon, S.: Ann. Scuol. Norm. Sup. Pisa, Ser. IV, Vol. II, 151-218 (1975)

2. Baeteman, M.L., Chadan, K.: Ann. Inst. H. Poincaré 24, 1-16 (1976)

3. Belopol'skii, A., Birman, M.Sh.: Izv. Akad. Nauk SSSR, Ser. Mat. 32, (1968)

4. Birman, M.Sh.: Mat. Sb. 55, 124-174 (1961)

5. Birman, M.Sh.: Vestn. Leningr. Univ. 13, 163-166 (1961)

6. Birman, M.Sh. : Izv. Akad. Nauk. SSSR, Ser. Mat. 32, 914-942 (1968)

7. Cotta-Ramusino, P., Krüger, W., Schrader, R.: Quantum scattering by external metrics and YangMills potentials. Freie Universität Berlin (preprint)

8. Combescure, M., Ginibre, J.: Ann. Inst. H. Poincaré 24, 17-30 (1976)

9. Combescure, M., Ginibre, J.: J. Funct. Anal. 29, 54-73 (1978)

10. Dollard, J., Friedman, C.: J. Math. Phys. 18, 1598-1607 (1977)

11. Dollard, J., Friedman, C.: Ann. Phys. 111, 251-266 (1978)

12. Enss, V.: Commun. Math. Phys. 61, 285-291 (1978)

13. Green, T., Lanford III, O.: J. Math. Phys. 1, 139-148 (1960)

14. Kato, T.: Math. Ann. 162, 258-279 (1966)

15. Kato, T.: J. Funct. Anal. 1, 342-369 (1967)

16. Kato, T.: Perturbation theory for linear operators, 2nd ed. Berlin, Heidelberg, New York: Springer 1976

17. Kuroda, S.T.: J. Anal. Math. 20, 57-117 (1967)

18. Lavine, R. : Indiana Univ. Math. J. 21, 643-656 (1972)

19. Matveev, V.B.: Teor. Math. Fiz. SSSR 15, 353-366 (1973)

20. Matveev, V.B., Skriganov, M.M.: Dokl. Akad. Nauk. SSSR, Ser. Mat., Fiz. 202, $755-758$ (1972)

21. Pearson, D.B.: Commun. Math. Phys. 40, 125-146 (1975)

22. Pearson, D.B. : Helv. Phys. Acta 48, 639-653 (1975)

23. Pearson, D.B.: Scattering theory for a class of oscillating potentials. Orsay preprint

24. Reed, M., Simon, B. : Methods of modern mathematical physics, Vol. I. New York: Academic Press 1973 
25. Reed, M., Simon, B.: Methods of modern mathematical physics, Vol. II. New York: Academic Press 1975

26. Reed, M., Simon, B.: Methods of modern mathematical physics, Vol. III. New York: Academic Press 1979

27. Reed, M., Simon, B.: Methods of modern mathematical physics, Vol. IV. New York: Academic Press 1978

28. Schechter, M. : Commun. Math. Helv. 49, 84-113 (1974)

29. Schwinger, J.: Proc. Natl. Acad. Sci. USA. 47, 122-129 (1961)

30. Simon, B.: Duke Math. J. 46, 119-168 (1979)

31. Skriganov, M.M.: Tr. Steklov. Math. Inst. 125, 187-195 (1973)

32. von Neumann, J., Wigner, E.P.: Z. Phys. 30, 465-467 (1929)

33. Ben Artzi, M., Devinatz, A. : J. Math. Phys. 20, 594-607 (1979)

34. Enss, V.: Ann. Phys. 119, 117-132 (1979)

Communicated by B. Simon

Received October 7, 1979; in revised form November 29, 1979 